\title{
Food Governance durch Qualitätszertifizierungen
}

\section{Rachel Reckinger, Diane Kapgen und Maria Helena Korjonen}

\section{Inhalt}

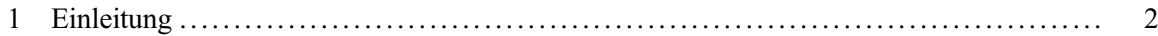

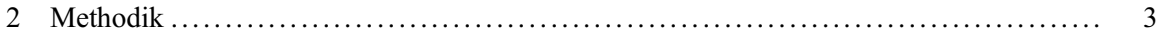

3 Kommunikationsstrategien von Lebensmittellabel in Luxemburg ................. 3

4 Kommunikative Anforderungen an Label zur Förderung von nachhaltigen

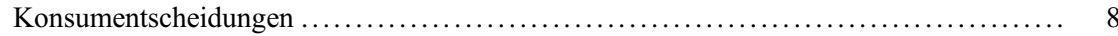

5 Wertschätzung von Lebensmitteleigenschaften $\ldots \ldots \ldots \ldots \ldots \ldots \ldots \ldots \ldots \ldots \ldots \ldots \ldots \ldots$

6 Öffentliches Beschaffungswesen als Chance zur Förderung von Nachhaltigkeit ........ 14

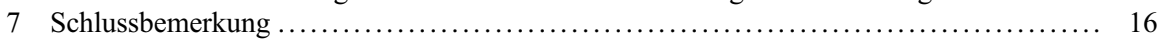

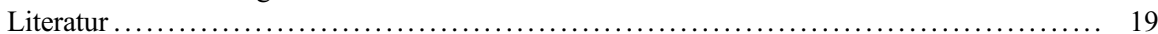

\section{Zusammenfassung}

In diesem Beitrag wird das Potential von Qualitätszertifizierungen zur Förderung eines nachhaltigen oder ethisch verantwortlichen Lebensmitteleinkaufs, sowohl in Bezug auf VerbraucherInnen als auch Akteure des öffentlichen Beschaffungswesens, untersucht. Es wird gezeigt, dass Governance-basierte Qualitätszertifizierungen wie Label effiziente Kommunikationsinstrumente für nachhaltigkeitsorientierte Ernährung sein können, indem sie Ernährungskompetenz und nachhaltigeres Einkaufsverhalten bei potentiell widersprüchlichen Interessen fördern, insbesondere, wenn die Zertifizierung den Mehrwert des Produktes transparent aufzeigt und die definierten Nachhaltigkeitskriterien gesetzlich verpflichtend sind.

\section{Schlüsselwörter}

Food Governance · Nachhaltiger Lebensmitteleinkauf · Luxemburgische Label · Qualitätszertifizierungen · Konsumentenverhalten

\footnotetext{
R. Reckinger $(\bowtie) \cdot$ D. Kapgen $\cdot$ M. H. Korjonen Department of Humanities, University of Luxembourg, Esch-sur-Alzette, Luxemburg E-Mail: rachel.reckinger@uni.lu; diane.kapgen@uni.lu; helena.korjonen@uni.lu
} 


\section{$1 \quad$ Einleitung}

Dieses Kapitel untersucht, ob Qualitätszertifizierungen in einem Umfeld zahlreicher und möglich widersprüchlicher Botschaften effiziente Governance-Instrumente der Ernährungskommunikation und einer verbesserten Ernährungskompetenz ${ }^{1}$ sein können. Dazu wird das Potential der Zertifizierungen analysiert, die die Priorisierung von Lebensmitteln mit nachhaltigem oder ethischem Mehrwert durch einzelne VerbraucherInnen und Akteure des öffentlichen Beschaffungswesens fördern. Als Grundlage dient der Vergleich verschiedener luxemburgischer Qualitätslabel. Es wird gezeigt, wie Label als Kommunikationsinstrument der Food Governance von den beiden wichtigsten Zielgruppen unterschiedlich genutzt werden: Privathaushalte nutzen Qualitätszertifizierungen aufgrund persönlicher und zum Teil verantwortunsgsbewusster Vorlieben als vertrauensbildende Zusatzinformation; Akteure des öffentlichen Beschaffungswesens nutzen Label in ihren Ausschreibungen vor allem aufgrund der politischen Maßgabe, Lebensmitteln mit nachhaltigem Mehrwert formal zu bevorzugen. Schließlich werden anhand von empirischen Daten Strategien von privaten KonsumentInnen zur Erlangung von Produktinformationen analysiert und herausgestellt, wie Produkteigenschaften von Qualitätszertifizierungen garantiert und von VerbraucherInnen wertgeschätzt werden. Bezüglich der Akteure des öffentlichen Beschaffungswesens wird der Blick darauf gerichtet, welche politischen Druckmittel zum Einsatz kommen, um die Nachfrage nach nachhaltigen Lebensmittelprodukten zu erhöhen.

Ernährungskommunikation wird hier als komplexer gesellschaftlicher Verständigungsprozess verstanden, der eine Fülle von Akteuren in verschiedenen gesellschaftlichen Teilsystemen und auf mehreren Entscheidungsebenen im Austausch von lebensmittelbezogenen Botschaften, Informationen, Wissensinhalten sowie Meinungen und Gefühlen vereint. In diesem Prozess des Austauschs ist Kommunikation ein primärer Prozess dynamischer und umstrittener Aushandlung der Realität (Cramer et al. 2011; LeBesco und Naccarato 2008; Godemann und Bartelmeß 2018). Dabei werden drei, sich in der gesellschaftlichen Praxis vermischende Formen der Ernährungskommunikation unterschieden: ${ }^{2}$ Kommunikation von Ernährung vollzieht sich durch das klassische, mono-direktionale (meist top-down) SenderEmpfänger-Modell, bei dem der Sender ein (verstecktes oder offenbartes) Kommunikationsziel verfolgt und der Empfänger die Botschaft eher passiv registriert. Kommunikation über Ernährung bezieht sich auf Prozesse, in denen Informationen,

\footnotetext{
${ }^{1}$ Das Verständnis von Ernährungskompetenz entspricht in diesem Beitrag dem von Vidgen und Gallegos (2014, S. 54): ,a collection of inter-related knowledge, skills and behaviors required to plan, manage, select, prepare and eat foods to meet needs and determine food intake. Food literacy is the scaffolding that empowers individuals, households, communities, and nations to protect diet quality through change, and support dietary resilience over time“.

${ }^{2}$ Zur Unterscheidung der drei Formen greifen wir auf aktuelle Debatten zur Nachhaltigkeitskommunikation (z. B. Newig et al. 2013) zurück und folgen deren Definitionen von „Kommunikation von, über und für Nachhaltigkeit". Andere AutorInnen wie Godemann und Bartelmeß (2018) verwenden die Klassifikation „Kommunikation über, von und durch Ernährung“.
} 
Interpretationen und Meinungen über Ernährung ausgetauscht und diskutiert werden, meist in horizontalen Kommunikationsströmen. Kommunikation für Ernährung setzt sich zusätzlich aktiv für einen Übergang zu nachhaltigen Ernährungspraktiken und -systemen ein, indem sie deren normative Ziele (d. h., wie sie aussehen sollen) fördert. Qualitätszertifizierungen schöpfen aus diesen verschiedenen Formen der Ernährungskommunikation, um verschiedene Akteure des Ernährungssystems zu verbinden und haben dadurch das Potential, die Entscheidungsfindung der Konsumierenden direkt zu beeinflussen.

\section{$2 \quad$ Methodik}

Der Beitrag stützt sich zum einen auf die Textanalyse veröffentlichter Dokumente sowie ,grauer' Literatur, die sich mit EU-weiten und nationalen Kennzeichnungssystemen $^{3}$ und der Vergabegesetzgebung in Luxemburg befasst. Diese Texte wurden mitels einer ,synoptischen qualitativen Inhaltsanalyse“ (Mayring 2007) ohne inhaltliche Verluste auf ihre wesentlichen Erkenntnisse verdichtet. Zum anderen wurden quantitative Erkentnisse aus einer repräsentativen Stichprobe der Wohnbevölkerung Luxemburgs und der umliegenden Grenzregionen ${ }^{4}(n=3300)$ aus dem Forschungsprojekt IDENT2 - Strategies of Regionalisation: Constructing Identity Across Borders (2011-2014; Wille et al. 2016) der Universität Luxemburg mit einbezogen.

\section{Kommunikationsstrategien von Lebensmittellabel in Luxemburg}

Trotz wachsendem Interesse der VerbraucherInnen an umwelt- und sozialverträglich hergestellten Produkten sowie der Anzahl nachhaltigkeitsbezogener Qualitätszertifizierungen (Janßen und Langen 2017) bleibt der Marktanteil von Lebensmitteln mit gesellschaftlichem oder ökologischem Mehrwert bisher gering (Rousseau und Vranken 2013). Qualitätslabel ${ }^{5}$ haben das Potential, diesen Anteil zu erhöhen, indem sie über die Leistungen der Produkte in Hinblick auf Nachhaltigkeit informieren (Nilsson et al. 2004). Auch wenn die gesetzliche Verpflichtung besteht, VerbraucherInnen

\footnotetext{
${ }^{3}$ Die freiwilligen EU-Richtlinien für Kennzeichnungssysteme ermöglichen es, einen methodischen Rahmen für die Analyse der empfohlenen Kennzeichnungskommunikation auf Grundlage der Kriterien Transparenz, Garantien und Kompetenzen zu schaffen.

${ }^{4}$ Für die Zwecke unserer Argumentation konzentrieren wir uns hier nur auf die in Luxemburg ansässigen Befragten $(n=1021)$.

${ }^{5}$ Hier wird der aus dem Englischen abgeleitete Oberbegriff Label verwendet, welcher verschiedene Informationssysteme, Marketinginstrumente und Zertifizierungsarten umfasst. Der im Deutschen landläufig verwendete Begriff Siegel wird bewusst vermieden, da er sich auf Produkte mit unabhängiger oder proprietärer Zertifizierung begrenzt, hier jedoch Produkte mit Eigenauskunft in die Analyse einbezogen werden.
} 
einen informativen Mehrwert zu gewährleisten, ${ }^{6}$ ist die Annahme, dass Label den verantwortungsvollen und nachhaltigen Konsum erhöhen würden, empirisch nicht belegt. Hinzu kommt, dass Label teilweise lediglich Marketingzwecken dienen, indem die gesellschaftliche bzw. ökologische Nachhaltigkeit der Produkte zum eigenen wirtschaftlichen Nutzen beschönigt wird (Clark und Russell 2004), und sie so zu einer Irreführung von VerbraucherInnen beitragen.

Die Analyse der Lebensmittellabel erfordert eine differenzierte Betrachtung von Labeltypen, den Eigentumsverhältnissen, den Eigenschaften der Label, den Kontrollmechanismen sowie der Lebensmittelkategorie (Nilsson et al. 2004). Durch diese Differenzierung wird deutlich, dass sowohl auf EU- als auch auf nationaler Ebene deutlich heterogene und ungleichmäßig formalisierte rechtliche Rahmenbedingungen existieren.

\subsection{Labeltypen: Prädominanz von nationalen und regionalen Label}

Von den in Luxemburg erfassten Label $^{7}$ dominieren ,Nationale Label` (44 \% der untersuchten Label), die Produkte kennzeichnen, die aus dem gesamten Staatsgebiet stammen. Es folgen ,Regionale Label‘ (25\%), die Produkte kennzeichnen, die aus einer bestimmten und abgegrenzten Region innerhalb Luxemburgs stammen. Das relativ ausgeprägte Phänomen ,regionaler Label' ist bemerkenswert für ein so kleines Land (2.586 qkm) ohne große biophysikalische Besonderheiten. ${ }^{8}$,Regionalität' wird also genutzt, um die Herkunft aus einem administrativ begrenzten Territorium zu kennzeichnen, und nicht aus einem biophysikalisch besonderen Territorium, wie beispielsweise im Sinne von Terroir. In einigen Fällen umfassen nationale und regionale Label auch Produkte aus der Großregion. ${ }^{9} \mathrm{Zu}$ den ,Biolabel' (16 \%) gehören Label, die entweder ausschließlich auf der EU-Ökoverordnung basieren (d. h., sie verwenden das EU-Biolabel) oder über die EU-Ökoverordnung hinausgehen (d. h., sie verwenden ein zusätzliches Biolabel). ,Supermarktlabel‘ (9\%) sind Label, die luxemburgischen Supermärkten gehören, was sie eher als Marken qualifizieren würde, doch da sie mit verbindlichen Lastenheften aufgestellt sind, können

\footnotetext{
${ }^{6}$ EU-Verordnung Nr. 1169/2011 über die Bereitstellung von Informationen über Lebensmittel für VerbraucherInnen.

${ }^{7}$ Insgesamt 32 Label, Stand der Labelerhebung, Dezember 2019. Siehe Anhang 1: Übersicht der Lebensmittellabel in Luxemburg nach Typen mit Angabe ihrer Hauptattribute, Zertifizierungsart und Eigentümerschaften.

${ }^{8}$ Luxemburg weist durchaus eine bemerkenswerte geologische Flächennutzungs- und Bodenvielfalt auf, jedoch nicht in dem Maße wie Regionen mit sehr besonderen Merkmalen (z. B. kontrastierende Bergregionen, die sich für die Kennzeichnung spezifischer Milchprodukte aus Bergqualität eignen).

${ }^{9}$ Definition der Großregion wie beschrieben in http://www.granderegion.net.
} 


\section{Eigentümerschaften der Labels}

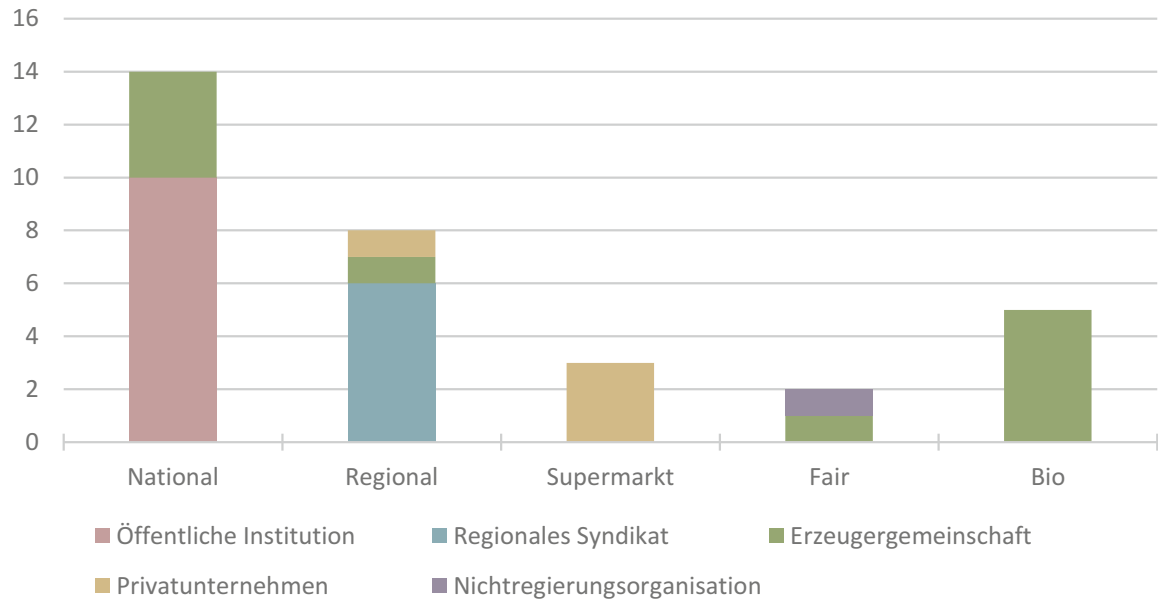

Abb. 1 Hauptinhaber luxemburgischer Labeltypen $(n=32)$

sie als Label angesehen werden. Die Haupteigenschaften der ,Fairen Label ${ }^{10}$ (6\%) sind die international gültigen Prinzipien des fairen Handels (s. Abb. 1).

Allgemein sind Lebensmittellabel am häufigsten im Besitz von Herstellern oder Einzelhändlern (Nilsson et al. 2004), in Luxemburg hingegen dominiert die Inhaberschaft von öffentlichen Institutionen (z. B. der Landwirtschaftskammer ${ }^{11}$ oder bestimmten Abteilungen innerhalb der Ministerien). Allein Biolabel sind ausschließlich im Besitz von Erzeugerverbänden (z. B. die Herdbuchgenossenschaft oder Bauernverbände), während Regionale Label in erster Linie im Besitz regionaler Syndikate (z. B. Naturparks oder interkommunale Kooperationseinrichtungen) sind und gleichzeitig die größte Vielfalt an Inhaberschaften aufweisen. Private Unternehmen (z. B. Supermärkte oder Schlachthöfe) und Nichtregierungsorganisationen (z. B. Fairtrade Luxemburg) sind seltener Eigentümer von Label.

Eine neuere, partizipativere Art der Inhaberschaft sind Multi-Stakeholder-Gremien, die aus der Erarbeitung der Labelattribute aus der ebenbürtigen Diskussion zwischen verschiedenen Akteuren, von den Produzenten über die Verarbeiter bis hin zu den EinzelhändlerInnen, idealerweise unter Einbeziehung von Akteuren der

\footnotetext{
${ }^{10}$ In diesem Kapitel nutzen wir die Begriffe „F/fair“, „Fair Trade“ und „fair gehandelt" als Synonyme, um Produkte zu beschreiben, die den international definierten Prinzipien des fairen Handels entsprechen und mit einem der zahlreichen international anerkannten Label für fairen Handel versehen sind. Wir nutzen den Begriff „Fairtrade“ ausschließlich und spezifisch für Produkte mit der „International Fairtrade Certification Mark“ von Fairtrade International (https://www. fairtrade.net/).

${ }^{11}$ Die Landwirtschaftskammer ist eine der fünf Berufskammern Luxemburgs.
} 


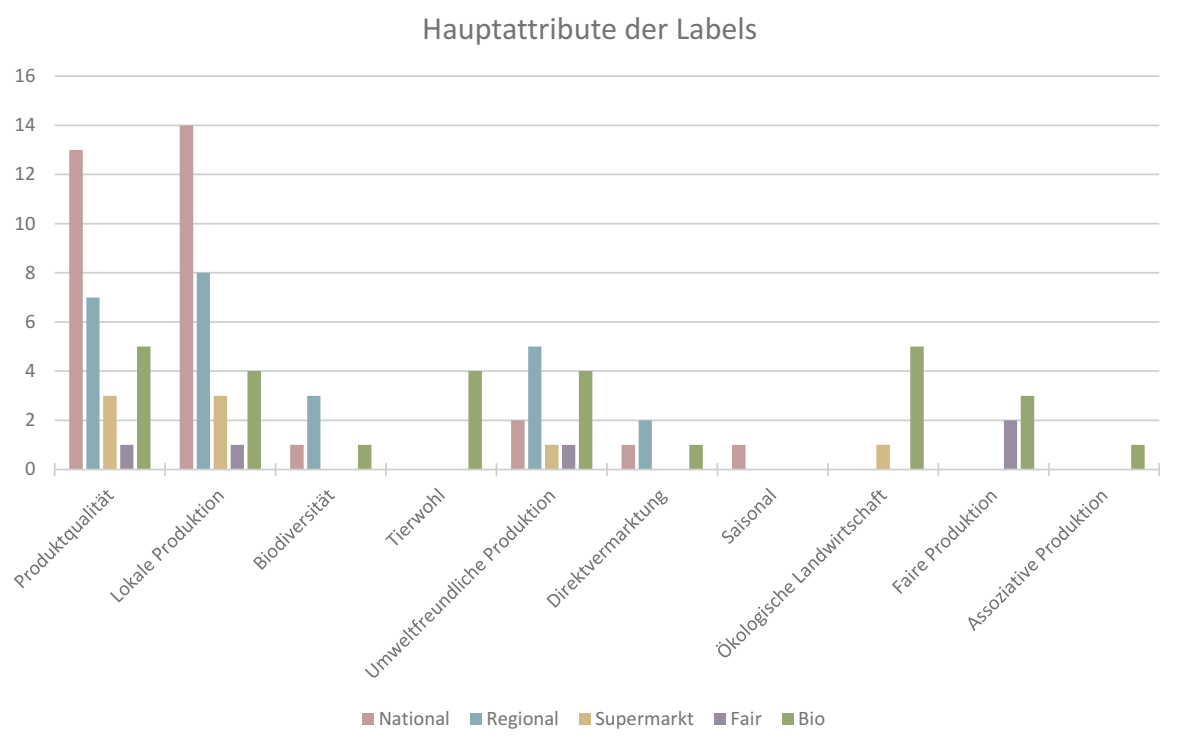

Abb. 2 Primäre Eigenschaften der Label Luxemburgs $(n=32)$

Zivilgesellschaft, hervorgehen. Beispielsweise verwendet die landesweite Biobauern-Kooperative BIOG partizipatorische Systeme mit Elementen der assoziativen Produktion.

In einigen Multi-Stakeholder-Systemen dominiert ein Akteur, daher ist das partizipatorische Element entsprechend schwächer ausgebildet. Dies lässt sich bei Hybridlabel mit Supermarktbeteiligung feststellen. Als stärkster Treiber von Hybridlabel entwickeln Supermärkte nicht nur eigene Bioprodukte, sondern schließen sich auch mit nationalen oder regionalen Label zusammen, um sich Exklusivrechte für den Verkauf ihrer Produkte zu sichern. Beispielsweise produziert das nationale Label Eist Uebst a Geméis Gemüse und Obst exklusiv für eine nationale Supermarktkette. Im Gegensatz zu Supermärkten fördern Label öffentlicher Institutionen nur selten Hybride, was den politischen Bemühungen zur ökologischen Agrarwende diametral gegenübersteht.

\subsection{Primäre Labeleigenschaften: Fokus auf Produktqualität und regionale Herkunft}

Aus den Lastenheften der Label lassen sich zehn primäre Eigenschaften der luxemburgischen Label ableiten, wie man in Abb. 2 sehen kann. 
Alle fünf Labeltypen beinhalten die Eigenschaften Produktqualität, lokale Produktion und - wenn auch in geringerem Maße - umweltfreundliche Produktion. Auffällig ist vor allem die Ausrichtung nationaler Label auf die ersten beiden Aspekte. Produktqualität ${ }^{12}$ als solche wird jedoch unterschiedlich interpretiert. Nationale Label im Besitz öffentlicher Institutionen verwenden Produktqualität im Sinne einer transparenten und regionalen Rückverfolgbarkeit des Produktes über die gesamte Lebensmittelwertschöpfungskette als Hauptrechtfertigung für die Kennzeichnung ihrer Produkte, und sind demnach wenig an Umwelt- oder Sozialkriterien gekoppelt. Darüber hinaus unterstreichen sie die hohe sensorische Qualität ihrer Produkte, insbesondere in ihrer Marketingkommunikation. Biolabel hingegen definieren Qualität als Ergebnis der landwirtschaftlichen Produktionsmethode (d. h. der ökologischen Landwirtschaft) und nicht als einen eigenständigen Wert, der sich aus der bloßen Rückverfolgbarkeit oder sensorischen Wahrnehmung ergibt.

Im Gegensatz zu nationalen fördern regionale Label eine größere Anzahl von unterschiedlichen Attributen gleichzeitig. Fairlabel dagegen legen vor allem Wert auf eine ,faire Produktion' und weniger auf andere Attribute. Biolabel haben insgesamt die umfassendsten Zielvorgaben. Bemerkenswert ist, dass faire Produktion öfter von Biolabel als von Fairlabel selbst vefolgt wird und dass das gesellschaftlich relevante Attribut , assoziative Produktion“ von keinem anderen Label verfolgt wird. Die Supermarktlabel spiegeln, wenn auch in einem recht geringen Maß, den Haupttrend des Schwerpunkts auf Produktqualität und Lokalität wider, gefolgt von Umweltfreundlichkeit sowie - durch ihre hybriden Konstellationen - Bioprodukten.

\subsection{Kontroverse um Zertifizierungsarten}

Label basieren auf zwei Zertifizierungsarten, die durch ihren Kontrollmechanismus definiert werden. In Luxemburg sind die meisten nationalen und Biolabel durch sogenannte Zertifizierungssysteme (CERT) geprüft, die (unabhängige) Bescheinigungsverfahren durch Dritte verwenden, während regionale und Supermarktlabel oft auf selbst erstellte Systeme (DEC) mit Selbstkontrollen durch den Inhaber oder durch nicht unabhängige (systemimminente) Prüfer zurückgreifen (Ipsos und London Economics Consortium 2013; Europäische Kommission 2010) (s. Abb. 3).

Der Kontrollmechanismus von Label ist jedoch zwiespältig. So kann ein Label vorgeben, dem Aspekt Tierschutz besonders verpflichtet zu sein, und genaue technische Details der Tierhaltungsbedingungen angeben. Auf diese Weise kann das Label ein Engagement für strenge unabhängige Kontrollen zum Tierwohl propagieren und diesen aufwertenden Anspruch an KundInnen weitergeben, obwohl es sich bei den Kontrollen tatsächlich um obligatorische Standardverfahren nach den EU-Rechtsvorschriften handelt, die die üblichen rechtlich festgelegten Aspekte des

\footnotetext{
${ }^{12}$ Allgemein ist Produktqualität ein polysemischer Begriff, der selten genau (innerhalb eines Labels) oder einheitlich (zwischen verschiedenen Label) definiert wird (Brunori 2007).
} 
Zertifizierungsart der Labels

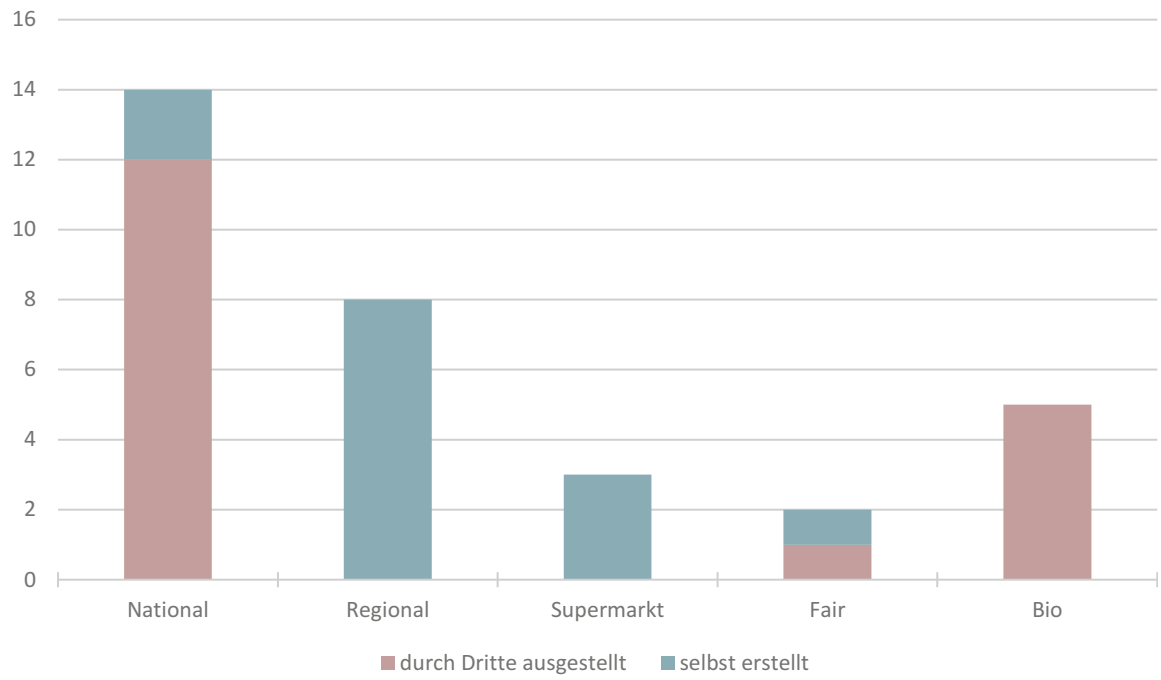

Abb. 3 Zertifizierung von Luxemburgs Labeltypen $(n=32)$

Tierwohls umfassen und nicht unbedingt einen zusätzlichen Beitrag des Labels zum Tierschutz bedeuten.

Während die Unterscheidung zwischen CERT- und DEC-Label eindeutig ist, führt eine externe Kontrolle nicht automatisch zu strengeren Label im Sinne ihres Beitrags zu nachhaltigeren Ernährungssystemen. Tatsächlich kann die Konnotation stärkerer Strenge, die mit unabhängigen externen Kontrollen einhergeht, die VerbraucherInnen zu der Annahme verleiten, dass alle vom Label aufgestellten Kriterien überprüft wurden, wobei unbekannt ist, inwieweit dies tatsächlich der Fall ist. Auch ist es möglich, dass kleinere Label mit selbst erstellten Zertifizierungen bedeutend über die gesetzlichen Mindestanforderungen hinausgehen, jedoch nicht über die finanziellen Mittel verfügen, um eine externe Zertifizierung durch Dritte durchzuführen.

\section{$4 \quad$ Kommunikative Anforderungen an Label zur Förderung von nachhaltigen Konsumentscheidungen}

Den Interpretationsspielraum, den das Konzept der Nachhaltigkeit mit sich bringt sowie das Fehlen präziser Vorschriften, was nachhaltige Lebensmittel kennzeichnet, lässt Qualitätszertifizierungen einen relativ großen Spielraum in der Nachhaltigkeitsinterpretation und -kommunikation. Der Blick auf Luxemburgs Labellandschaft zeigt, dass Lebensmittelkennzeichnungssystemen meist über ihren mutmaßlichen ökologischen, sozialen und gesundheitlichen Nutzen kommunizieren, während die wirtschaftlichen Vorteile für die Labelinhaber verborgen bleiben. Gleichzeitig ist oft 
intransparent, wem das Label gehört, welche Angaben kontrolliert werden und ob die Kriterien bezüglich ökologischer, sozialer oder gesundheitlicher Vorteile über die gesetzlichen Vorgaben hinausgehen. Folglich können VerbraucherInnen den versprochenen Mehrwert der Produkte nicht ohne Weiteres überprüfen (Vega-Zamora et al. 2019; Wang et al. 2017). Letztlich haben VerbraucherInnen nur dann eine tatsächliche Garantie für den spezifischen Mehrwert durch Label, wenn deren Inhaber eine dreifache Kommunikation für Ernährung betreiben: erstens eine vollständige Angabe aller für die aufgestellten Ansprüche geltenden Gesetze und Vorschriften; zweitens eine detaillierte Erläuterung, inwiefern die einzelnen, klar definierten Labelattribute über diese Gesetze hinausgehen; und drittens eine Offenlegung, welche dieser Attribute durch Dritte kontrolliert oder selbst deklariert werden.

Im folgenden Teil wird aufgezeigt, wie private VerbraucherInnen die Eigenschaften, die die Label garantieren, bewerten und wie sie auf diese Informationen zugreifen.

\section{$5 \quad$ Wertschätzung von Lebensmitteleigenschaften}

Allgemeine Prioritäten von VerbraucherInnen beim Lebensmitteleinkauf sind Saisonalität (67\%), gefolgt vom Preis (64\%), regionaler Herkunft (51\%) und ökologischer Landwirtschaft (30 \%) (Reckinger 2016, 2020). Da lokale Produktion und biologische Landwirtschaft zu den Hauptattributen von Qualitätszertifizierungen zählen, werden diese beiden Attribute hier näher untersucht, zusammen mit dem des fairen Handels und dem der Saisonalität, sowie dem subjektiv-pragmatischen, aber (haus-)wirtschaftlichen Attribut der Preiswertigkeit. Auf diese Attribute konzentriert sich auch die Entscheidungsfindung im öffentlichen Beschaffungswesen (SICONA-Ouest \& SICONA-Centre 2016).

Die folgenden Abbildungen, die auf einer Matrix verschiedener Lebensmittel basieren, veranschaulichen die subjektive Bedeutung detaillierter, die VerbraucherInnen den genannten ethischen und wirtschaftlichen Attributen beimessen. ${ }^{13}$

\subsection{Lebensmittelwahl}

Für spezifische Lebensmittel wird Regionalität ${ }^{14}$ von VerbraucherInnen als wichtigstes Kriterium bewertet, gefolgt vom Preis und zuletzt der Herkunft aus ökologischem Anbau. Regionale Herkunft wird bei Fleisch und Wurstwaren (78 \%),

\footnotetext{
${ }^{13}$ „Ethisch“ meint, dass die Wahl eines der Attribute eine bewusste und nach den eigenen ethischen Kategorien der VerbraucherInnen ausgerichtete Entscheidung erfordert; sie impliziert nicht automatisch, dass Regionalität, Saisonalität oder ökologische Landwirtschaft anderen Alternativen ethisch überlegen seien, vgl. die Kritik an der „local trap“, die fälschlicherweise davon ausgeht, dass der lokale Maßstab der Nahrungsmittelproduktion an sich bereits Qualität garantiert (Born und Purcell 2006).

${ }^{14}$ Regionalität ist eine spezifische und territorial abgegrenzte Form allgemeiner geografischer Herkunft (die hauptsächlich nachvollziehbar sein muss; Reckinger 2016, S. 255).
} 


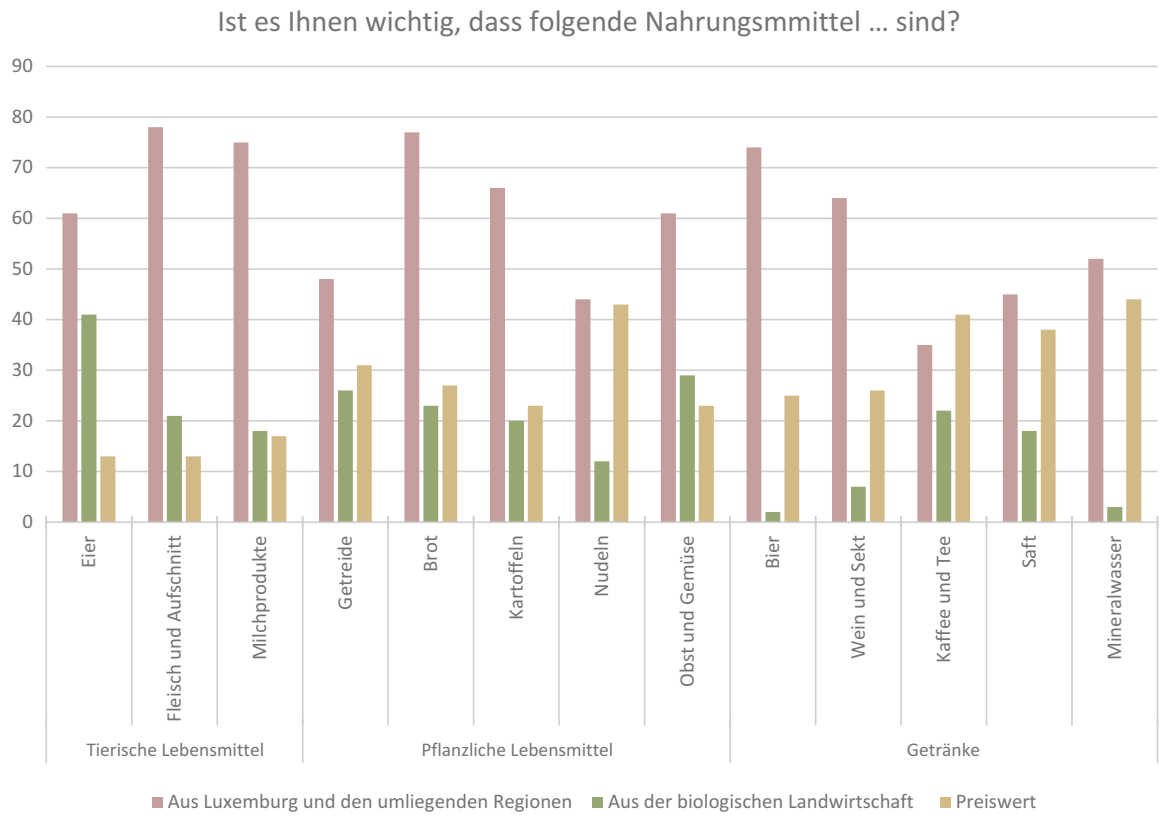

Abb. 4 Ist es Ihnen wichtig, dass folgende Nahrungsmittel [Aus Luxemburg und den umliegenden Regionen/Aus der biologischen Landwirtschaft/Preiswert] sind? („Umgebende Regionen“ sind alltagspragmatisch als ein $50 \mathrm{~km}$ langer Gürtel um die nationalen Grenzen Luxemburgs definiert, d. h. ein Ansatz, der sich von offiziellen administrative-territorialen Einheiten unterscheidet (Wille et al. 2016))

Brot (77\%), Milchprodukten (75 \%) und Bier (74 \%) am höchsten bewertet. Es folgen Kartoffeln (66 \%), Wein und Sekt (64\%), Eier sowie Obst und Gemüse (jeweils $6 \%$ ). Eine lokale Herkunft wird also im Allgemeinen für alltägliche Grundnahrungsmittel als wünschenswert angesehen. Nur bei Kaffee und Tee wird Regionalität vom Preis übertrumpft. Bei Teigwaren sind Regionalität und Preis den VerbraucherInnen gleichermaßen wichtig. Ein günstiger Preis spielt generell beim Einkauf von Mineralwasser, Teigwaren und Fruchtsäften eine größere Rolle. Bei tierischen Erzeugnissen hingegen schätzen VerbraucherInnen die Herkunft aus ökologischer Landwirtschaft höher als einen Billigpreis: insbesondere bei Eiern sowie bei Fleisch und Wurstwaren ist diese Tendenz ausgeprägt, bei Milchprodukten eher schwächer. Die einzige andere Lebensmittelkategorie, bei der die Herkunft aus ökologischer Landwirtschaft wichtiger als der Preis angesehen wird, sind Obst und Gemüse. Generell wird der ökologische Anbau am wichtigsten erachtet bei Eiern (41\%) und am niedrigsten bei Bier (2\%) (s. Abb. 4).

Die Herkunft aus fairem Handel spiegelt anscheinend für die VerbraucherInnen die höchste Bedeutung bei Produkten aus den klassischen Interventionsbereichen der internationalen Label im Bereich des fairen Handels wider, vor allem solche, die sich an ErzeugerInnen und Erzeugerkooperativen in Entwicklungsländern richten: Kaffee und Tee sowie exotisches Obst und Gemüse (s. Abb. 5). 
Ist es Ihnen wichtig, dass folgende Nahrungsmittel aus dem

\section{fairen Handel sind?}

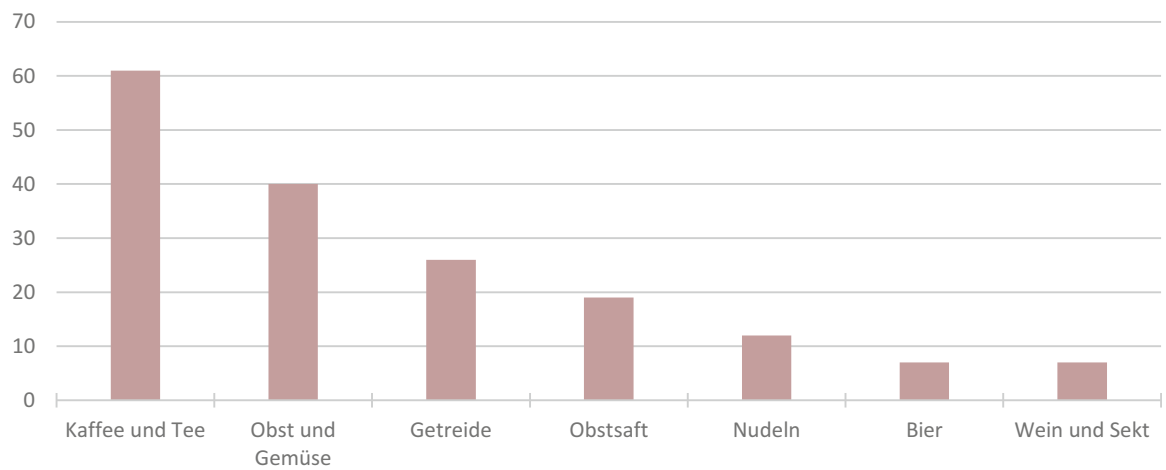

Abb. 5 Die Bedeutung, die einzelne VerbraucherInnen dem fairen Handel mit Lebensmitteln beimessen

Was tun Sie, um herauszufinden, ob ein Lebensmittel aus Luxemburg und den umliegenden Regionen kommt / saisonal ist / aus der biologischen Landwirtschaft ist / aus dem fairen Handel ist?

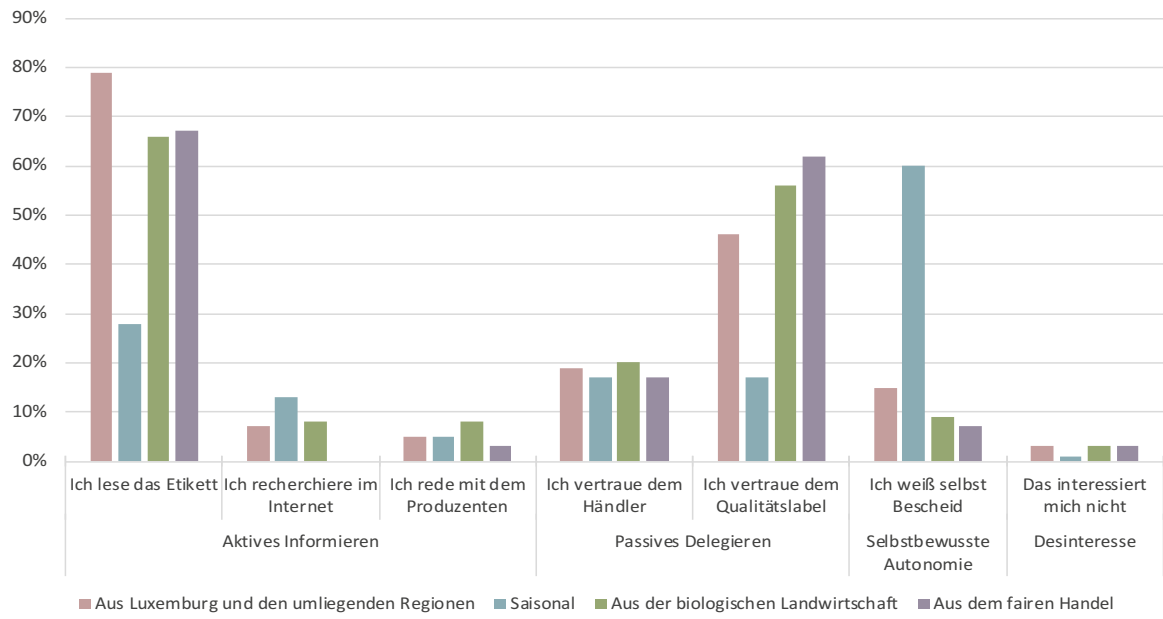

Abb. 6 Möglichkeiten der VerbraucherInnen, sich über die Herkunft von Lebensmitteln zu informieren (\%)

\subsection{Informationsmöglichkeiten}

Um Informationen über die Eigenschaften von Lebensmitteln zu erlangen, wenden VerbraucherInnen verschiedene Strategien an: aktive Informationssuche, passive Delegierung, selbstbewusste Autonomie sowie Desinteresse (s. Abb. 6). 
Insgesamt nutzen VerbraucherInnen nicht die ganze Bandbreite möglicher Informationsquellen, sondern neigen dazu, mehrere Bricolage-Techniken zu kombinieren (Reckinger und Régnier 2017). Für Produkte aus regionaler, biologischer oder fairer Herkunft sind die zwei am häufigsten angewandten Strategien: „Ich lese das Etikett“ - am häufigsten bei regionalen Produkten (79 \%), gefolgt von fair gehandelten (67 \%) und Bioprodukten (66 \%) - und „Ich vertraue dem Qualitätslabel“ - am häufigsten bei fairen Produkten (62\%), gefolgt von Bioprodukten (56 \%) und regionalen Produkten (46\%). Hierbei ist Ersteres eine aktive Informationsbeschaffung, die direkt am Kaufort erfolgt, möglicherweise in Bezug auf einzelne Produkte, nicht unbedingt vorsätzlich und eher Teil eines kumulativen Lern- und Spezialisierungsprozesses. Zweiteres ist hingegen eine passive Delegierung des Vertrauens, ${ }^{15}$ mit oder ohne vorherige Information über die Spezifikationen bzw. Garantien, die vom Label auf dem Etikett geliefert werden. Diese Strategie bezieht sich auf eine Reihe von Produkten von bereits identifizierten und eingeprägten Marken und nicht auf einzelne Lebensmittel, was auch das hohe Vertrauen in faire Label zeigt: Sie werden international transversal kommuniziert, sind daher bekannt und eingängig zu verstehen.

Im Fall der Saisonalität von Obst und Gemüse (Reckinger 2020) ${ }^{16}$ sind die Ergebnisse im Vergleich zu den anderen drei Attributen völlig unterschiedlich. Zwei Drittel der Befragten geben an „Ich weiß selbst Bescheid“ (60 \%). Nur ein Drittel

\footnotetext{
${ }^{15}$ Eine eingehende Analyse des Verbrauchervertrauens würde den Rahmen dieses Artikels überschreiten, aber wir können feststellen, dass es verschiedene Formen des Vertrauens gibt, die von den VerbraucherInnen am Punkt der informierten Entscheidungsfindung praktiziert werden, z. B. funktionales Vertrauen, das auf der Überzeugung beruht, dass ein Produkt bestimmte Eigenschaften hat oder mehr Vorteile bietet als ein anderes Produkt und somit seine Funktion erfüllt (Vega-Zamora et al. 2019); systemisches Vertrauen in die Lebensmittelindustrie, ihre Sicherheit sowie die Authentizität und Integrität ihrer Produkte, wie es durch wahrheitsgetreue Etiketten und ihre Garantien für Produktionsmethoden, Zertifizierungsverfahren und -stellen, Etikettierungs- und Verkaufskontrolle vermittelt wird (Vega-Zamora et al. 2019); aber auch das Vertrauen zwischen VerbraucherInnen und VerkäuferInnen/ProduzentInnen, das oft auf Bauernmärkten und Veranstaltungen aufgebaut wird, bei denen VerbraucherInnen direkten Zugang zu ProduzentInneen und VerkäuferInnen haben (Savadori et al. 2007; Prigent-Simonin und Hérault-Fournier 2005).

${ }^{16}$ Wie schon einige Jahre zuvor gaben die Befragten, wenn sie mithilfe eines Fragebogens befragt werden, erneut eine Präferenz für saisonales Obst und Gemüse an: $82 \%$. Zwar geht dies über den Rahmen dieses Kapitels hinaus, aber wir haben an anderer Stelle gezeigt, dass diese enorme Tendenz der sozialen Erwünschtheit nicht durch qualitative Daten untermauert wird, in denen die Saisonalität nur selten spontan erwähnt wird, und wenn dann als diätetische, im Wesentlichen pädagogische Norm oder als moralischer Zwang (Reckinger 2020).
} 
liest das Etikett und nur $17 \%$ vertrauen dem Label. Das Vorhandensein von Vorkenntnissen und informalem Wissen ist hier auffällig. Dennoch geben $28 \%$ der Befragten an, dass sie auf den Produktetiketten nach Informationen zur Saisonalität suchten. Dies zeugt von einer reflexartigen Erwartungshaltung bzgl. dem Informationsgehalt von Etiketten oder deutet darauf hin, dass KonsumentInnen sich eine solche explizite Kommunikation auf Etiketten wünschen würden (wobei ein Ampelsystem - kombiniert mit einem vereinfachten Saisonkalender auf der Produktverpackung - durchaus vorstellbar ist). Vorerst können die EtikettenleserInnen also nur über die obligatorische Herkunftsangabe Vermutungen zur Saisonalität anstellen: Kommt etwa der Spargel im Februar aus Peru, ist er in Westeuropa wahrscheinlich noch nicht in der Saison. Der Mangel an Informationen auf dem Etikett und das gleichzeitige Fehlen von Label, die die Saisonalität bescheinigen, wird in dem Fall durch andere Strategien der Informationsbeschaffung kompensiert. So vertrauen bei der Saisonalität nur $17 \%$ der Befragten ihren HändlerInnen durch passive Delegierung. Zudem wird bei der Saisonalität im Vergleich zu den anderen Attributen am meisten im Internet recherchiert. Dies ist eine aktive, jedoch nicht auf die unmittelbare Kaufsituation beschränkte Geste, die potentiell zu einer kumulativem Lernleistung und Spezialisierung führt.

Informationsbeschaffungsstrategien von VerbraucherInnen unterscheiden sich bei der Saisonalität von regionaler Herkunft, fairem Handel und ökologischer Landwirtschaft: Bei der Saisonalität wird vor allem informales Vorwissen genutzt, während bei den anderen Attributen eine $A d$-hoc-Mischung aus Informationssuche durch flüchtiges Etikettenlesen und passivem, generischem Vertrauen in eventuelle Labelabbildungen auf dem Etikett angewendet wird. „Ich vertraue dem Einzelhändler“ ist für alle Produktkategorien bemerkenswert einheitlich, was zeigt, dass die Marketingstrategien von Supermärkten eigenständige Marken und Label zu entwerfen, eine erfolgreiche Kommunikationsleistung sind.

Das Biolabel, das von allen Label die umfassendste Einbeziehung von gleichzeitig ökologischen und sozialen Kriterien garantiert, hat für private VerbraucherInnen keine zentrale Priorität (Reckinger 2020). Hingegen haben VerbraucherInnen eine klare Präferenz für regionale Lebensmittel - ein Trend, der sich auch in der positiven Entwicklung regionaler Label sowie in der Priorisierung der öffentlichen Beschaffung beobachten lässt. Im folgenden Teil wird aufgezeigt, wie das öffentliche Beschaffungswesen in Luxemburg Label als kommunikative Kaufentscheidungshilfen nutzt. 
 \\ Öffentliches Beschaffungswesen als Chance zur Förderung von Nachhaltigkeit}

Die öffentliche Beschaffung ${ }^{17}$ von Nahrungsmitteln bietet eine Gelegenheit, die Ernährungswirtschaft auf nachhaltigere Wege zu lenken, denn durch die Einbeziehung sozialer, ökologischer und ökonomischer Erwägungen kann sie umfassende Vorteile für Umwelt, Gesellschaft und Wirtschaft haben (Soldi 2018). Im öffentlichen Beschaffungswesen wird die Bewertung der Produkte in Form von funktionalem Vertrauen in die Überlegenheit eines Produkts gegenüber einem anderen sowie in Form von systemischem Vertrauen in die allgemeine Integrität und Authentizität eines Produkts in die Hände von Fachleuten in der öffentlichen Beschaffung gelegt. Dies zwingt das öffentliche Beschaffungswesen zur Transparenz und dazu, Garantien für das öffentliche Wohl zu bieten, kurzum sich als vertrauens- und glaubwürdig zu präsentieren. Qualitätszertifizierungen für Lebensmittel sind in dem Zusammenhang vertrauensbildende Kommunikationsinstrumente, da sie den KonsumentInnen signalisieren, dass das öffentliche Beschaffungswesen Produkte auswählt, die eine überdurchschnittliche Qualitätszertifizierung nachweisen - zusätzlich zum geltenden Qualitätsstandard in der Lebensmittelbeschaffung. Darüber hinaus bieten sie formalisierte politische Ansatzpunkte, um öffentliche Einkäufe rechtlich auf Lebensmittel mit ethischem und nachhaltigem Mehrwert auszurichten. Diese Ausrichtung wird auch von politischen Richtlinien unterstützt. Auf EU-Ebene spielt das freiwillige EU-Instrument der umweltfreundlichen öffentlichen Beschaffung (Green Public Procurement, GPP) eine Schlüsselrolle in den Bemühungen, eine kritische Masse zur Nachfrage nach nachhaltigeren Gütern und Dienstleistungen anzuregen.

Auf nationaler Ebene existieren einige innovative - zum Teil partizipatorisch gestaltete - Richtlinien und Initiativen, um die Nachfrage nach Produkten und Dienstleistungen, die auf lokalen, saisonalen, biologischen oder sozialen Kriterien basieren, in der öffentlichen Beschaffung zu fördern. Nachfolgend werden die wichtigsten dieser Initiativen auf ihre Nachhaltigkeitskriterien analysiert, wobei die EU-Richtlinien für eine umweltfreundliche öffentliche Beschaffung im Lebensmittel- und Gaststättengewerbe EU Food \& Catering Services Product Brief for Green Public Procurement (Europäische Kommission 2012) den analytischen Rahmen bilden. Diese Richtlinien unterscheiden zwischen grundlegenden und zusätzlichen Kriterien für die Beschaffung von Lebensmitteln und -dienstleistungen. Die grundlegenden Kriterien beziehen sich auf ökologische Produktionsmethoden sowie Abfallmeidung und -verwertung; zusätzliche Kriterien betreffen Umweltaspekte (Umweltmanagementsysteme, Schulung des Personals, umweltpolitische Empfehlungen und Umweltbewusstsein der Lieferanten). Die Auswahl von Obst und Gemüse sollte saisonal erfolgen, und alle Produkte durch ein Biolabel oder ein ähn-

\footnotetext{
${ }^{17}$ Das öffentliche Beschaffungswesen für Lebensmittel umfasst Bildungs-, Gesundheits- und Sozialeinrichtungen, Büros, Kantinen, Sport- und Freizeitzentren, staatliche und öffentliche Einrichtungen, Catering-Dienste usw. In diesem Beitrag liegt der Fokus auf Bildungseinrichtungen, da die öffentliche Ernährungskommunikation in diesem Bereich am stärksten formalisiert wurde.
} 
liches nationales, etabliertes Label verifiziert sein. Basierend auf dieser Richtlinien hat der luxemburgische Staat den Leitfaden Catering - Service de la restauration (Tudor 2014) $^{18}$ erstellt, der EinkäuferInnen aus dem öffentlichen Beschaffungswesen helfen soll, nach Nachhaltigkeitskriterien zu planen, sowie zwischen nationalen und EU-Label (z. B. Bio-Lëtzebuerg, Demeter oder dem EU-Biolabel) sowie denen aus Nachbarländern (Frankreich und Deutschland) zu vergleichen, da Einkauf und Konsum auch grenzüberschreitend stattfinden.

Spezifisch für die öffentliche Beschaffung von Lebensmitteln in Bildungseinrichtungen existieren einige interessante zusätzliche Empfehlungen. Beispielsweise hat das interkommunale Syndikat SICONA, den Leitfaden Anforderungen an den Einkauf von nachhaltig produzierten Lebensmitteln für ,,Maisons Relais“. Textbausteine zur Ergänzung von Lastenheften der Gemeinden (SICONA-Ouest und SICONACentre 2016) herausgegeben, der Empfehlungen für den Einkauf von Lebensmitteln durch die Maison Relais ${ }^{19}$ in kommunaler Verantwortung ${ }^{20}$ ausspricht. Die Empfehlungen sollen ,eine regionale, saisonale und faire sowie wünschenswerte biologische Versorgung “ der Schulküchen gewährleisten, sind jedoch weder obligatorisch noch rechtlich bindend. Die Empfehlungen sind Teil eines umfassenderen Projektes zur Förderung der regionalen Landwirtschaft, das Landwirte einer bestimmten Region verpflichtet, qualitativ hochwertige Lebensmittel gemäß bestimmter Vorgaben (entweder in biologischer oder konventioneller Qualität) zu produzieren. Das Erreichen eines Höchstmaßes an Qualitätskriterien wird durch eine Abwägung der Punkte pro Leistungskriterium gefördert. Dieses System ermöglicht Optimierungen in der Produktionskette innerhalb jedes beitragenden Betriebs sowie eine Vergleichbarkeit der Produkte von ökologischen und konventionellen Betrieben (ökologische Betriebe erzielen tendenziell höhere Punktzahlen). Die Maison Relais aus derselben Region werden anhand spezieller Kaufempfehlungen ermutigt, diese Produkte, sowohl ökologische als auch konventionelle, in ihren Beschaffungsstrategien zu bevorzugen. Diese Kaufempfehlungen bedienen sich eines Ampelcodes, um zu verdeutlichen, welche Arten von Lebensmitteln sich für den Kauf anbieten (Abb. 7).

\footnotetext{
${ }^{18}$ Basierend auf dem EU Food \& Catering Services Product Brief for Green Public Procurement wurde es entwickelt als Teil eines Projekts zur Umsetzung der Ziele des Luxemburger nationalen Nachhaltigkeitsplans (https://environnement.public.lu/fr/actualites/2018/septembre2018/pndd.html).

${ }^{19}$ Ausserschulische, öffentliche Betreuungsstrukturen für Kinder im Kindergarten- und Grundschulalter, die oftmals an die Schulgebäude angegliedert sind.

${ }^{20}$ Die öffentliche Beschaffung von Nahrungsmitteln in den Bildungseinrichtungen Luxemburgs wird im Fall der öffentlichen Vorschuleinrichtungen und Grundschulen dezentral von den 102 Gemeinden des Landes geleitet. Demgegenüber wird die Lebensmittelbeschaffung im Sekundarund Hochschulbereich zentralisiert von Restopolis, dem 2005 gegründeten Schul- und Universitätsverpflegungsdienst des Ministeriums für nationale Bildung, Kinder und Jugend, betreut.
} 


\begin{abstract}
Abb. 7 Ampelsystem für Kaufempfehlungen für Maison Relais (SICONAOuest und SICONA-Centre 2016, S. 3)
\end{abstract}

\begin{tabular}{|l|}
\hline „Natur genéissen“-Produkte \\
\hline Regionale Produkte \\
\hline Produkte mit regionalem Bezug \\
\hline Weitere Produkte (Produkte ohne regionalen Bezug) \\
\hline Zu vermeidende Produkte \\
\hline
\end{tabular}

Ausgehend von einer hierarchischen Staffelung der Faktoren Regionalität, Umweltschutz, Fairtrade sowie der landwirtschaftlichen Produktionsmethode ${ }^{21}$ werden unterschiedlich gekennzeichnete Produkte und bestimmte Label empfohlen.

Um Nachhaltigkeit im öffentlichen Beschaffungswesen zu fördern, wurde zudem das geltende Gesetz revidiert. Es erleichtert explizit die Teilnahme kleiner und mittlerer Unternehmen (KMU), indem kleinere öffentliche Aufträge ermöglicht werden. Das Gesetz erhebt außerdem den Anspruch, das öffentliche Beschaffungswesen nachhaltiger zu gestalten, indem es gewichtete (z. B. qualitative, soziale oder ökologische, normen- oder managementbezogene) Kriterien festlegt und so die Auswahl zwischen verschiedenen Qualitätslabel und Zertifizierungen erleichtert. Zusätzlich zu den schon genannten Initiativen wollen die Empfehlungen der Chambre des Métiers für die Versorgung des öffentlichen Marktes mit Nahrungsmitteln die nachhaltige Entwicklung der Regionen unterstützen, indem die regionale Nahrungsmittelproduktion gefördert und so die Umweltbelastung der Produkte durch lange Transportwege minimiert wird (Chambre des Métiers 2017).

Die vorgestelltenVeränderungen im öffentlichen Beschaffungswesen sind sowohl eine Bottom-up-Antwort auf das wachsende Interesse der VerbraucherInnen an regionalen und nachhaltigen Lebensmitteln und für eine bessere Transparenz bei der Lebensmittelbeschaffung als auch eine Top-down-Verpflichtung im Bereich der Lebensmittelpolitik, um die Beschaffung ressourceneffizienter zu gestalten und die Nachfrage nach nachhaltigeren Gütern und Dienstleistungen anzuregen. Letztlich handelt es sich bei den vorgestellten Initiativen um „Best-Practice-Beispiele“, die neue Wege beschreiten und (noch) nicht alle gesetzlich verankert sind. Sie schlagen Lösungen vor, die über das geltende Lebensmittelbeschaffungsrecht hinausgehen und - wenn sie einmal erprobt und bewährt sind - als Vorbilder für künftige nachhaltigere gesetzliche Rahmenbedingungen dienen könnten.

\section{$7 \quad$ Schlussbemerkung}

In einem Umfeld, in dem LebensmittelproduzentInnen und EinzelhändlerInnen zunehmend gezwungen sind, auf die Nachhaltigkeitsdebatte zu reagieren, haben Qualitätszertifizierungen bzw. Label das Potential, ein Hebel für nachhaltigere

\footnotetext{
${ }^{21}$ Es wird empfohlen, Produkte zu mindestens $30 \%$ aus biologischer und maximal $70 \%$ aus konventioneller Produktion zu beziehen.
} 
Ernährungssysteme zu sein, indem sie eine Kommunikation für Ernährung betreiben, nicht zuletzt durch eine Verbesserung der Ernährungskompetenz und ein einhergehendes nachhaltigeres Kauf- oder Beschaffungsverhalten - sowohl für private Haushalte als auch für Akteure des öffentlichen Beschaffungswesens. Qualitätszertifizierungen machen sich dabei die Tatsache zunutze, dass sich der Lebensmittelkonsum nicht auf den Kauf eines Produkts beschränkt, sondern in einem zugrundeliegenden Sinn- und Wertesystem fußt (LeBesco und Naccarato 2008). Es besteht jedoch das Risiko, dass Marketing- oder Markenstrategien mit rein wirtschaftlichen Zielen als eine solche Kommunikation für Ernährung getarnt werden. Doch selbst bei asymmetrischen Kommunikationsprozessen und der Schwierigkeit, Behauptungen zu verifizieren, können VerbraucherInnen das Potential von Qualitätszertifizierungen nach ihren persönlichen Prioritäten (Verbeke 2008) und ihren Strategien der Informationssuche abschätzen. Zur Informationssuche nutzen VerbraucherInnen unterschiedliche Bricolage-Techniken. Was ihre Prioritäten betrifft, so sind VerbraucherInnen in Luxemburg vor allem die Regionalität und der Preis wichtig - aber weit weniger die ökologische Produktion. Die relativ geringe Priorität von Bioprodukten in privaten Haushalten mag die Schwierigkeiten widerspiegeln, mit denen Biolabel in ihrer Kommunikation für nachhaltige Ernährungssysteme konfrontiert sind: Während normative Nachhaltigkeitsziele am konsequentesten durch Biolabel umgesetzt werden (mithilfe ihrer umfassenden Einbeziehung ökologischer und sozialer Kriterien), werden die Prioritäten der VerbraucherInnen weiterhin von anderen Realitäten geprägt, die nicht unbedingt im Einklang mit Nachhaltigkeitsbemühungen stehen. In der öffentlichen Beschaffung hingegen werden Bioprodukte und andere in Label formalisierte Qualitätskriterien stetig verpflichtender, in dem politischen Bestreben, im Land erzeugte Lebensmittel mit Mehrwert in verschiedenen Ethikbereichen zu begünstigen. In einem Umfeld vielfältiger und potentiell widersprüchlicher Botschaften können Governance-basierte Qualitätszertifizierungen ihr Kommunikationspotential für Ernährung im Endeffekt nur freisetzen, wenn sie ihren Mehrwert transparent aufzeigen (statt ihn nur zu beteuern) und, wenn sie durch Gesetze unterstützt werden, die definierte und anspruchsvolle Nachhaltigkeitskriterien verbindlich vorschreiben (statt sie nur zu empfehlen). 
Anhang 1 Übersicht der Lebensmittellabel in Luxemburg nach Typen mit Angabe ihrer Hauptattribute, Zertifizierungsart und Eigentümerschaften

\begin{tabular}{|c|c|c|c|c|c|c|c|c|c|}
\hline \multicolumn{10}{|c|}{ NATIONAL } \\
\hline \multirow{3}{*}{$\begin{array}{l}\begin{array}{l}\text { Marque Nationale' } \\
\text { Beurre } \\
\text { (Beurre rose) }\end{array} \\
\text { एत्र }\end{array}$} & Zertifizierung & $\begin{array}{l}\text { Marque Nationale } \\
\text { Miel }\end{array}$ & Zertifizierung & $\begin{array}{l}\text { Marque Nationale } \\
\text { Jambon }\end{array}$ & Zertifizierung & $\begin{array}{l}\text { Marque Nationale } \\
\text { Viande de Porc }\end{array}$ & Zertifizierung & $\begin{array}{l}\text { Marque Nationale } \\
\text { Eau de Vie }\end{array}$ & Zertifizierung \\
\hline & $\begin{array}{l}\text { Produktqualiät, } \\
\text { Lokale Produktion }\end{array}$ & & $\begin{array}{l}\text { Produktqualität, } \\
\text { Lokale Produktion }\end{array}$ & & $\begin{array}{l}\text { Produktaualitiat, } \\
\text { Lokale Produktion }\end{array}$ & & $\begin{array}{l}\text { Produktqualität, } \\
\text { Lokale Produktion }\end{array}$ & $\approx$ & $\begin{array}{l}\text { Produktqualität, } \\
\text { Lokale Produktion }\end{array}$ \\
\hline & Öffentliche Institution & & Öffentliche Institution & & Öffentliche Institution & & Öffentliche Institution & & Öffentliche Institution \\
\hline \multirow{3}{*}{$\begin{array}{l}\text { Produit du Terroir } \\
\text { - Lëtzzebuerger } \\
\text { Rêndfleesch }\end{array}$} & Zertifizierung & $\begin{array}{l}\text { Produit du Terroir } \\
\text { - Lêtzebuerger }\end{array}$ & Zertifizierung & $\begin{array}{l}\text { Produit du Terroir } \\
\text { - Lëtzebuerger }\end{array}$ & Zertifizierung & $\begin{array}{l}\text { AOP Moselle } \\
\text { Luxembourgeoise }\end{array}$ & Zertifizierung & $\begin{array}{l}\text { Made in } \\
\text { Luxembourg }\end{array}$ & Zertifizierung \\
\hline & $\begin{array}{l}\text { Produktqualitït, } \\
\text { Lokale Produktion }\end{array}$ & 送 & $\begin{array}{l}\text { Produktqualität, } \\
\text { Lokale Produktion }\end{array}$ & a Brout & $\begin{array}{l}\text { Produktqualität, } \\
\text { Lokale Produktion }\end{array}$ & TE & $\begin{array}{l}\text { Produktqualität, } \\
\text { Lokale Produktion }\end{array}$ & గాల్ & $\begin{array}{l}\text { Produktqualität, } \\
\text { Lokale Produktion }\end{array}$ \\
\hline & Öffentliche Institution & & Offentliche Institution & & Öffentliche Institution & & Erzeugergemeinschaft & & Öffentliche Institution \\
\hline \multirow{3}{*}{$\begin{array}{l}\text { Sou schmaacht } \\
\text { Lëtzebuerg }\end{array}$} & Zertifizierung & $\begin{array}{l}\text { Naturschutz- } \\
\text { fleesch }\end{array}$ & Zertifizierung & $\begin{array}{l}\text { Eist Uebst } \\
\text { a Geméis }\end{array}$ & Eigenerklärung & $\begin{array}{l}\text { Vum Bauerenhaff } \\
\text { op den Dësch }\end{array}$ & Eigenerklärung & & \\
\hline & $\begin{array}{l}\text { Produktqualität, } \\
\text { Lokale Produktion }\end{array}$ & 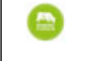 & $\begin{array}{l}\text { Produktqualität, } \\
\text { Biodiversitatit, } \\
\text { Tierwohl }\end{array}$ & $d:=$ & $\begin{array}{l}\text { Umweltfreundliche } \\
\text { Produktion, lokale } \\
\text { Produktion, Saisonal, } \\
\text { Produktqualităt }\end{array}$ & (3) & $\begin{array}{l}\text { Lokale Produktion, } \\
\text { Direktvermarktung }\end{array}$ & & \\
\hline & öffentliche Institution & & Erreugergemeinschaft & & Erzeugergemeinschaft & & Erzeugergemeinschaft & & \\
\hline \multicolumn{10}{|c|}{ REGIONAL } \\
\hline \multirow{3}{*}{ Vêi vum Séi } & Eigenerklärung & Téi vum Séi & Eigenerklärung & Spelz vum Séi & Eigenerklärung & Gebäck vum Séi & Eigenerklärung & Bléi vum Séi & Eigenerklärung \\
\hline & $\begin{array}{l}\text { Lokale Produktion, } \\
\text { Produktqualität, } \\
\text { Umweltfreundliche } \\
\text { Produktion }\end{array}$ & ina & $\begin{array}{l}\text { Lokale Produktion, } \\
\text { Produktqualitatt, } \\
\text { Umweltfreundliche } \\
\text { Produktion }\end{array}$ & ares & $\begin{array}{l}\text { Lokale Produktion, } \\
\text { Produktqualität, } \\
\text { Umwelffreundliche } \\
\text { Produktion }\end{array}$ & conka & $\begin{array}{l}\text { Lokale Produktion, } \\
\text { Produktqualität, } \\
\text { Umwelffreundliche } \\
\text { Produktion }\end{array}$ & BLÉI ج्र्र & $\begin{array}{l}\text { Lokale Produktion, } \\
\text { Produktqualität, } \\
\text { Umweltfreundliche } \\
\text { Produktion }\end{array}$ \\
\hline & Regionales Syndikat & & Regionales Syndikat & & Regionales Syndikat & & Regionales Syndikat & & Regionales Syndikat \\
\hline \multirow{3}{*}{ Natur Geneissen } & Eigenerklärung & Ourdaller & Eigenerklärung & Eisleker Gourmet & Eigenerklärung & & & & \\
\hline & $\begin{array}{l}\text { Lokale Produktion, } \\
\text { Umweltfreundliche } \\
\text { Produktion }\end{array}$ & and & $\begin{array}{l}\text { Produktqualitït, } \\
\text { Lokale Produktion }\end{array}$ & inten & $\begin{array}{l}\text { Produktqualitït, } \\
\text { Lokale Produktion }\end{array}$ & & & & \\
\hline & Regionales Syndikat & & Erzeugergemeinschaft & & Privatunternehmen & & & & \\
\hline
\end{tabular}

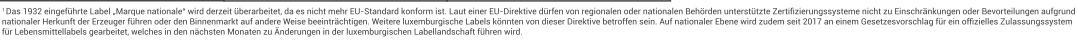

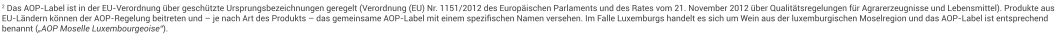

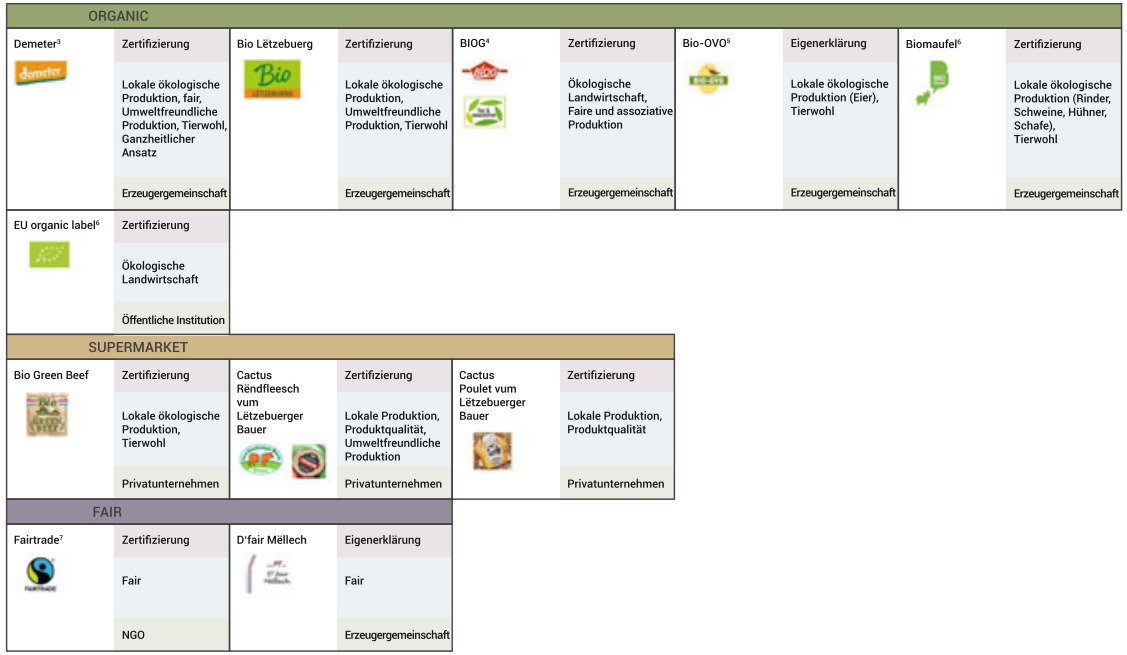

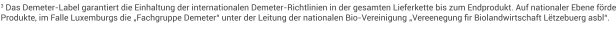

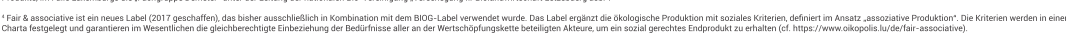
"Bib-ovo ist tim Begifif, bis Mitte 2020 ein von Dritten zertifiziertes Label 7 w werden.

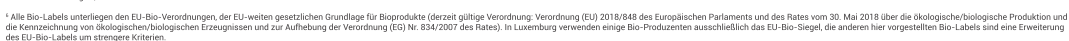




\section{Literatur}

Born, B., \& Purcell, M. (2006). Avoiding the local trap. Journal of Planning Education and Research, 26(2), 195-207. https://doi.org/10.1177/0739456X06291389.

Brunori, G. (2007). Local food and alternative food networks: A communication perspective. Anthropology of Food, (S. 2). https://doi.org/10.4000/aof.430.

Chambre des Metiers. (2017). Plateforme „marché public" - rubrique fourniture de denrées alimentaires. http://nobe.lu/uploads/pdfs/Examples/Alimentation/PlateformeMDDIMarch éspublics20170508_final.pdf. Zugegriffen am 13.10.2020.

Clark, C. D., \& Russell, C. S. (2004). Ecolabels and economic efficiency: some preliminary results. American Agricultural Association Annual Meeting. https://ageconsearch.umn.edu/record/ 20338/files/sp04c101.pdf. Zugegriffen am 13.10.2020.

Cramer, J. M., Greene, C. P., \& Walters, L. (2011). Food as communication: Communication as food. New York: Peter Lang.

European Commission. (2010). Commission communication - EU best practice guidelines for voluntary certification schemes for agricultural products and foodstuffs. https://eur-lex.europa. eu/LexUriServ/LexUriServ.do?uri=OJ:C:2010:341:0005:0011:en:PDF.

European Commission. (2012). Alimentation et services de restauration Fiche Produit pour les marchés publics écologiques (MPE). Mallette de formation de la Commission européenne sur les MPE - Module 3: Recommandations d'achat.

Godemann, J., \& Bartelmeß, T. (2018). Gesellschaftliche Verständigung über ein Totalphänomen. Zum Verständnis nachhaltigkeitsbezogener Ernährungskommunikation. In T. Phyel (Hrsg.), Zwischen Ohnmacht und Zuversicht? Vom Umgang mit Komplexität in der Nachhaltigkeitskommunikation (S. 187-206). München: oekom.

Ipsos \& London Economics Consortium. (2013). Consumer market Study on the functioning of voluntary food labelling schemes for consumers in the European Union EAHC/FWC/2012 8604.

Janßen, D., \& Langen, N. (2017). The bunch of sustainability labels - Do consumers differentiate? Journal of Cleaner Production, 143, 1233-1245. https://doi.org/10.1016/j.jclepro.2016.11.171.

LeBesco, K., \& Naccarato, P. (2008). Edible ideologies: Representing food and meaning. New York: State University of New York Press.

Mayring, P. (2007). Qualitative Inhaltsanalyse. Grundlagen und Techniken. Weinheim: Beltz.

Newig, J., Schulz, D., Fischer, D., Hetze, K., Laws, N., Lüdecke, G., \& Rieckmann, M. (2013). Communication regarding sustainability: Conceptual perspectives and exploration of societal subsystems. Sustainability (Switzerland), 5(7), 2976-2990. https://doi.org/10.3390/su5072976.

Nilsson, H., Tunçer, B., \& Thidell, §̊. (2004). The use of eco-labeling like initiatives on food products to promote quality assurance - Is there enough credibility? Journal of Cleaner Production, 12(5), 517-526. https://doi.org/10.1016/S0959-6526(03)00114-8.

Prigent-Simonin, A.-H., \& Hérault-Fournier, C. (2005). The role of trust in the perception of the quality of local food products: With particular reference to direct relationships between producer and consumer. Anthropology of Food, 4. https://doi.org/10.4000/aof.204.

Reckinger, R. (2016). Sustainable everyday eating practices from the perspective of spatial identifications. In C. Wille, R. Reckinger, S. Kmec \& M. Hesse (Hrsg.), Spaces and identities in border regions. Politics - Media - Subjects (S. 252-266). Bielefeld: transcript.

Reckinger, R. (2020). Reprise de conscience de la saisonnalité via la régionalité. In L'alimentation au fil des saisons. La saisonnalité des pratiques alimentaires (S. 71-92). Rennes: Presses Universitaires de Rennes.

Reckinger, R., \& Régnier, F. (2017). Diet and public health campaigns: Implementation and appropriation of nutritional recommendations in France and Luxembourg. Appetite, 112, 249-259. https://doi.org/10.1016/j.appet.2017.01.034.

Rousseau, S., \& Vranken, L. (2013). Green market expansion by reducing information asymmetries: Evidence for labeled organic food products. Food Policy, 40, 31-43. https://doi.org/ 10.1016/j.foodpol.2013.01.006. 
Savadori, L., Graffeo, M., Bonini, N., Lombardi, L., Tentori, K., \& Rumiati, R. (2007). Rebuilding consumer trust in the context of a food crisis. In M. Siegrist, T. C. Earle \& H. Gutscher (Hrsg.), Trust in risk management: Uncertainty and scepticism in the public mind (S. 159-171). London: Earthscan. https://www.researchgate.net/publication/264382950_Rebuilding_consumer_trust_ in the context_of a food_crisis. Zugegriffen am 13.10.2020.

SICONA-Ouest \& SICONA-Centre. (2016). Anforderungen an den Einkauf von nachhaltig produzierten Lebensmitteln für ,,Maisons Relais “. http://nobe.lu/uploads/pdfs/Examples/Alimenta tion/SICONA_Lastenheft_Naturgenéissen_180416.pdf. Zugegriffen am 13.10.2020.

Soldi, R. (2018). Committee of the Regions \& Progress Consulting S.r.l. (n.d.). Sustainable public procurement of food. https://op.europa.eu/en/publication-detail/-/publication/b1b7d65b-533411e8-be1d-01aa75ed71a1/language-en/format-PDF/source-114398136.

Tudor, H. (2014). Catering - Service de la restauration. http://nobe.lu/uploads/pdfs/Examples/ Alimentation/Tudor_Marches_publics_durables.pdf. Zugegriffen am 13.10.2020.

Vega-Zamora, M., Torres-Ruiz, F. J., \& Parras-Rosa, M. (2019). Towards sustainable consumption: Keys to communication for improving trust in organic foods. Journal of Cleaner Production, 216, 511-519. https://doi.org/10.1016/j.jclepro.2018.12.129.

Verbeke, W. (2008). Impact of communication on consumers' food choices. Proceedings of the Nutrition Society, 67(3), 281-288. https://doi.org/10.1017/S0029665108007179.

Vidgen, H. A., \& Gallegos, D. (2014). Defining food literacy and its components. Appetite, 76, 50-59. https://doi.org/10.1016/j.appet.2014.01.010.

Wang, C. S., Van Fleet, D. D., \& Mishra, A. K. (2017). Food integrity: A market-based solution. British Food Journal, 119(1), 7-19. https://doi.org/10.1108/BFJ-04-2016-0144.

Wille, S., Reckinger, R., Kmec, S., \& Hesse, M. (Hrsg.). (2016). Spaces and identities in border regions. Politics - Media - Subjects. Bielefeld: transcript. 